\title{
Neurobiology of PTSD and implications for treatment: An overview
}

\author{
Maria-Olga Sakellariou and Athena Stefanatou*
}

Sakellariou MO, Stefanatou A. Neurobiology of PTSD and implications for treatment: An overview. Curr Res Integr Med 2017;2(1):6-9.

Recent respective research findings in Neuroscience unfold a scientific breakthrough in the understanding of the neurobiology of psychological trauma and its immediate and long term consequences. By shedding light on the connection between biological processes and human experience and behavior when trauma occurs, they conduce hopefully to identify important options for treatment.
Due to the extensiveness and complexity of PTSD we have concentrated our elaboration mostly drawing upon meta-analysis studies as a source. A combination of medication and psychotherapy is suggested for the treatment of PTSD. Body awareness studies are promising. As scientists and clinicians, we certainly have to develop methods of treating a disease as a personal experience of a global phenomenon. The traumatic experience remains a personal story to be told.

Key Words: Post-traumatic stress, Neurobiology of PTSD, Treatment of PTSD
$\mathrm{T}$ raumatic events accompany our everyday life in the form subjective painful losses and wounding: It may be the loss of a home pet, the loss of a job, robbery, a car accident, natural disasters, severe illness, the death of a beloved person, emotional neglect and abuse, betrayal, bullying, sexual abuse, rape, an earthquake, war, a terrorist attack. According to statistics one out of two persons will be exposed to a life-threatening event during lifetime (1). Some of them will be able to overcome this experience; some will be severely affected in the long term. Relevant studies indicate that about 7\% of Americans have a prevalence to develop a Post-Traumatic Stress Disorder (PTSD) at some point of their lives. According to statistical data men are more often exposed to traumatic events than women, but women are more likely to suffer from a PTSD disorder $(2,3)$. Respective data for Greece are at this time not available. Nevertheless, the refugees' crisis we are facing as a hosting country arises the issue of PTSD to a first line social problem that we have to face in terms of policy measures and treatment options. How can Neurobiology contribute to the understanding of what happens in body and mind of a person suffering from PTSD? Can neurobiological findings reveal a new perspective in the treatment of PTSD beyond pharmacotherapy and classical psychotherapeutic approaches that might contribute to develop effective psychotherapeutic interventions? What does the experience of PTSD mean for the offspring of PTSD sufferers?

\section{From a traumatic experience to PTSD}

Extreme stressful situations in life are addressed by an instinctive "fight or flight" response in order to assure survival. Alerting sensory environmental information reaches the brain and enters the amygdala, the operation center of the brain in the case of extreme stressful situations that is responsible for the activation of the hypothalamic - pituitary adrenal axis (HPA) and the sympathetic nervous system (SNS). This activation produces multiple effects in body and mind in order for the individual to cope with the stressors. Via the SNS system the adrenal glands release hormones called catecholamines into the blood causing increased blood pressure, glucose levels and heart rate in order to support the body to "fight or fly". At the same time the hypothalamus, when stimulated releases neuropeptides that lead to the discharge of cortisol that binds the glucocorticoid receptors in the brain and reduces stress responses (4). Usually the stress response function contributes to the adaptation to threat and restores the homeostasis of the organism with an extinction of these reactions when stimuli are not present any more. Traumatic experiences are triggered by perceived overwhelming danger and are accompanied by intense feelings of fear, horror and helplessness. Such situations may lead to a dysregulation of the described underlying biological stress mechanisms known as the "fear circuitry" leading to stress related symptoms like PTSD. The state of chemical dysregulation is also named "allostatic load" (5). If a person is able to recover after a traumatic event or this event becomes disruptive and destructive for his life even though the threat has passed, depends on several external and internal factors, the later also known as "neurobiological markers for PTSD" (6). The nature, severity, duration and recurrence of traumatization as well as the persons' own heredity, childhood, medical history and resilience are decisive on his/ her ability to process physiologically and psychologically the experience. Processing and interpreting the event in a meaningful manner have an impact on whether the experience can be integrated by the person in his/her narrative or whether it causes great physiological and psychological damage.

Developmental trauma is one of the most important risk factors for PTSD. In the present study, it will not be addressed in detail, although we are aware of the fact that it's occurrence in childhood leads to deficient organization and enduring dis-function of the right hemisphere in regulatory issues and to disorganized or broken attachment bonds. For the purpose of this study we consider it a fact that early developmental trauma leads to maladaptive infant health that decreases its resilience and resources and increases the probability of developing PTSD as a maladaptive coping mechanism in adult life (7).

In the following we will focus on PTSD as a possible long term response to extreme traumatic experiences with devastating functional impairment and will search for the underlying neurobiological processes.

\section{Definition of trauma and the DSM-V}

Trauma is defined as a stressful response to serious unusual life altering and/ or life threatening events that when consolidated, disrupts time in before and after and has chronically enduring effects, even though the threat has passed. These effects might last throughout life and even persist to the next generation. As a variety of clinical phenotypes are consistent with the phenomenology of PTSD a decision was made in the DSM V to no longer consider PTSD as an anxiety disorder and attach it to the new category "Trauma and stress related disorders".

As in the introduction of this category is mentioned "Psychological distress following exposure to a traumatic or stressful event is quite variable.... Many individuals who have been exposed to a traumatic or stressful event exhibit a phenotype in which, rather than anxiety- or fear-based symptoms, the most prominent clinical characteristics are an- hedonic and dysphoric symptoms, externalizing angry and aggressive symptoms, or dissociative symptoms (DSM-V, p.302).

Post-traumatic stress disorder according to the DSM-V (26) is diagnosed when a person (Criteria paraphrased):

A. Is confronted with death, threatened death, actual or threatened serious injury, or actual or threatened sexual violence via direct or indirect exposure or by witnessing or by being exposed to aversive details of the event due to professional duties (Criterion A rephrased). 
B. Suffers from intrusion of symptoms in the form of recurrent, intrusive involuntary recollections of the traumatic event in the form of nightmares, dissociative reactions in the form of flashbacks or even unconsciousness, intense distress and physiological reactions after exposure to traumatic triggers (Criterion B rephrased, one out of five symptoms needed).

C. Avoids persistently and with great effort distressing stimuli like thoughts or feelings and external reminders (places, persons, music etc.) that are connected to the traumatic event (Criterion $\mathrm{C}$ rephrased, one out of two symptoms needed)

D. Experiences negative alterations in mood and cognition, both starting and getting worst after the event: Dissociative amnesia, Negative beliefs and expectations about oneself, distorted blame of self or others, feelings of fear, horror, anger, shame, diminished interest in meaningful activities, feelings of alienation from others and constriction of affect (Criterion D rephrased, two out of seven symptoms needed).

E. Shows alterations in arousal and reactivity in association to the traumatic event as irritable or aggressive behavior, self-destructive or reckless behavior, hypervigilance, exaggerated startle response, problems in concentration and sleep disturbance (Criterion E rephrased, two out of six symptoms needed)

F. Persistence of symptoms in the Criteria B,C,D,E for longer than one month after the event

G. Significant distress or impairment that is related to the symptoms

$\mathrm{H}$ Previously mentioned symptoms do not occur due to other medical condition, substance abuse or medication

The DSM V introduces for adult and children the Dissociative Subtype of PTSD where the PTSD diagnostic criteria are met and concurrently high levels of depersonalization or de - realization are being experienced.

\section{Neurobiology of PTSD}

The abovementioned signs and symptoms might reflect a persevering adaptation of the underlying neurobiological mechanisms to cope with life-threatening extreme situations. Recent research studies offer a very complex picture of the neurobiological dynamic of PTSD. They focus on the exploration of different aspects of neurobiological changes in PTSD sufferers in regard to endocrinology, neurochemistry and brain circuitry and on the linkage that these changes might have to the clinical features of PTSD as hyperarousal, Impulsivity, dysregulated executive functions and memory $(6,8-11)$.

\section{Endocrine factors}

PTSD is characterized by abnormal regulation of hormones in the hypothalamic pituitary adrenal axis, the center of the mammalian neuro endocrine stress response. When exposed to stress, neurons in the hypothalamic ventricular nucleus (PVN) secrete Corticotrophin releasing hormone $(\mathrm{CRH})$ that stimulates the production and release of Adreno Corticotrophin (ACTH) into the anterior pituitary. Glucocorticoids are stimulated by ACTH and released by the adrenal cortex. Glucocorticoids are the central modulator of metabolism, brain and immune functionality in order to coordinate the physiological management of stress. ACTH is transported to the adrenal gland where the hormone cortisol is produced. Cortisol activates via the Locus Coeruleus and the Norepinephrine system (NE) the Sympathetic Nervous System (SNS) and gives negative feedback to both the hypothalamus and the anterior pituitary.

At the same time neurons in different brain areas modulate the HPA axis activity (defined as a complex set of direct influences and feedback interactions among three endocrine glands: the hypothalamus, the pituitary gland, and the adrenal glands.) having an increasing effect (amygdala and brain stem) and an inhibiting effect (hippocampus and prefrontal cortex) on Corticotrophin releasing hormone (CRH). Glucocorticoid exposure of the hippocampus leads to reduction of dendritic branching, loss of spines and decreased neurogenesis. The activation of the HPA axis in uncontrollable stress - a situation related to the traumatic experience - that threatens the physical integrity has a high, flat diurnal profile of cortisol release (with lowerthan-normal levels of cortisol in the morning and higher-than-normal levels in the evening) resulting in a high overall level of daily cortisol release (12).

In regard to the endocrine factors it can be concluded that the activation of the HPA axis leads to sustained increased levels of Corticotrophin releasing hormone (CRH) and at the same time to "Hypocortisolism" as detected in studies of urine and blood of combat veterans with PTSD compared with healthy controls and other groups as, survivors of holocaust, refugees and abused persons (13). In terms of behavior these changes may have an effect on oversensitive response to stress, abnormal stress encoding and fear processing. Furthermore, PTSD has been associated with NR3C1 epigenetic modifications that were similarly found in the mothers and their offspring, modifications that may underlie the possible transmission of biological alterations of the HPA axis (Figure 1) (14).

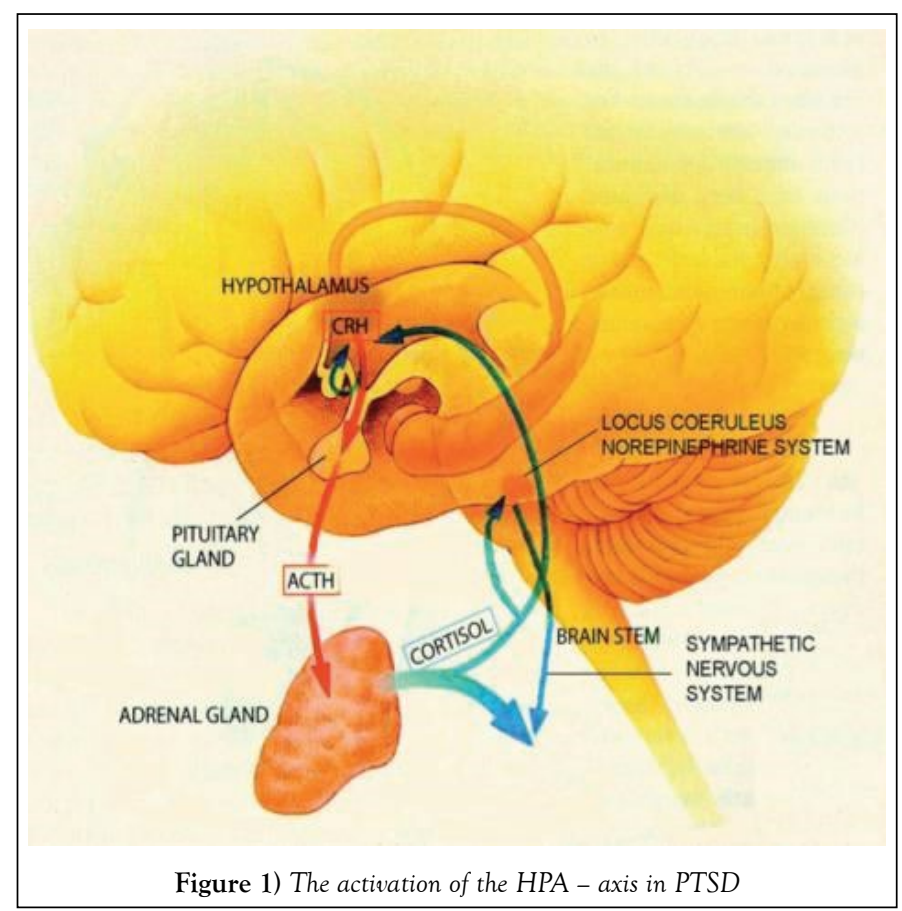

\section{Neurochemical factors in PTSD}

In regard to neurochemicals by PTSD abnormal regulation was found in catecholamine, serotonin, amino acids, peptide and opioid neurotransmitters, all of them having a regulating and integrating function for fear and stress responses in the brain circuitry (Figure 2).

By PTSD sufferers in recent research studies there have been found increased levels of the catecholamines Dopamine (DA) and Norepinephrine (NE) that may be responsible for the physiological effects as increased pulse and blood pressure, startle response and levels of arousal. They have a negative effect on fear conditioning, encoding of and response to fear memories.

Serotonin $(5 \mathrm{HT})$ originates in the brainstem and projects, having a homeostatic role, to different brain areas such as the amygdala, the hippocampus and the prefrontal cortex that are crucial in the processing of traumatic experiences. According to studies (6) decreased Serotonin levels were identified in PTSD sufferers that may disturb the dynamic relationship between amygdala and hippocampus, abolishing its capacity to reduce anxiety and contributing to increased vigilance, impulsivity and intrusion of memories.

GABA, an amino acid, is a neurotransmitter with inhibitory influence in the brain. According to several studies (6) decreased GABA activity was found by PTSD sufferers thus explaining their restricted ability to moderate physiological reactions to stress and anxiety. On the other hand Glutamate, the primary excitatory neurotransmitter in the brain that binds to $\mathrm{N}$ methyl D-aspartate receptors (NMDA) is responsible for the consolidation of memories. It was found to have increased levels by PTSD sufferers thus contributing to the consolidation of trauma memories, de - realization reactions and disassociation phenomena.

Corticotrophin (CRH) and Norepinephrine (NE) neurons, both neuropeptides have an increased activity thus contributing to exaggerated stress responses, numbing, analgesia and disassociation.

\section{Brain circuitry in PTSD}

By using brain imaging techniques in research characteristic changes have been identified in the structure of certain brain areas that might be related to impaired functioning of PTSD sufferers $(8,6)$. Especially the Hippocampus, the amygdala and the anterior cingulate cortex (ACC), insula and orbitofrontal region (OFC) in the prefrontal Cortex (PFC) are affected. (Table 1) connects structural and functional changes in the respective brain areas with the impaired physiological/behavioral function. 
TABLE 1

PTSD relevant brain areas, structural changes and effects on behavior

\begin{tabular}{|c|c|c|}
\hline Brain region & Change & $\begin{array}{l}\text { Impaired physiological/ } \\
\text { behavioural Function }\end{array}$ \\
\hline Hippocampus & & $\begin{array}{c}\text { Exaggerated activation and } \\
\text { inability to terminate stress } \\
\text { response, deficits in verbal } \\
\text { memory }\end{array}$ \\
\hline \multirow{2}{*}{$\begin{array}{c}\text { Para-hippocampal } \\
\text { gyrus }\end{array}$} & $\begin{array}{l}\text { Reduced volume and } \\
\text { plasticity }\end{array}$ & \\
\hline & & $\begin{array}{l}\text { Impaired extinction of fear } \\
\text { conditioning \& non-discrimination } \\
\text { between safe/unsafe }\end{array}$ \\
\hline Amygdala & $\begin{array}{c}\text { No clear evidence for } \\
\text { structural alterations } \\
\text { but increased } \\
\text { reactivity }\end{array}$ & $\begin{array}{l}\text { Hyper responsiveness to } \\
\text { stressful stimuli but also to } \\
\text { neutral stimuli as faces }\end{array}$ \\
\hline
\end{tabular}

$\begin{array}{cc}\text { Medial Prefrontal } & \text { Decreased volume of } \\ \text { Cortex (PFC) } & \text { PFC, ACC and OFC } \\ & \text { correlated with the } \\ \text { severity of symptoms }\end{array}$

Decreased reactivity of PFC to exert inhibitory control over stress responses, decreased emotional reactions by OFC and ACC (numbing)

Anterior cingulate cortex (ACC)

Under functioning

Decreased sensory awareness and bodily integration

\begin{tabular}{ccc} 
Insula & Under functioning & \\
\hline Sensorimotor Cortex evidence for & $\begin{array}{c}\text { structural alterations } \\
\text { but increased } \\
\text { reactivity }\end{array}$ & $\begin{array}{c}\text { Hyperarousal when traumatic } \\
\text { stimulus exposure }\end{array}$ \\
\hline
\end{tabular}

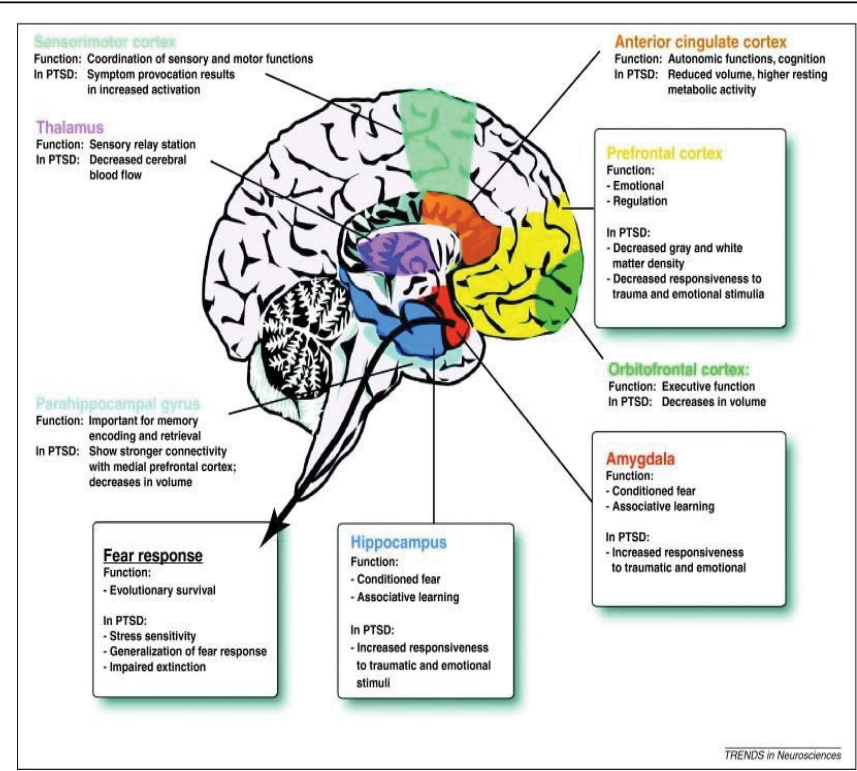

Figure 2) PTSD relevant brain areas, functionality and PTSD impairment

Trans-generational transmission of trauma in offspring of Holocaust, combat veterans and genocide survivors is an issue that attracted in the last decades the interest of the trauma focused psychotherapist, mostly from a family systems perspective that perceives transmission from a relational point of view: that perpetuates contagious, repeated and observable patterns of communication within the family $(15,16)$. Alternative approaches describe the identified PTSD symptoms in second and third generation offspring of survivors of Holocaust as an adaptation of family values and beliefs following the family's historical track and its disruption (17) and not as a pathological reaction to the trauma of previous generations.

In her neurobiological research, Yehuda addresses the question of epigenetic mechanisms to PTSD (18) where an "epigenetic modification refers to a change in the DNA produced by an environmental perturbation that alters the function but not the structure of a gene" (p.428) that can be transmitted intergenerationally. She proposes an epigenetic model illustrated by glucocorticoid gene methylation where an environmental exposure alters the function not the structure of the gene, which influences the sub consequent reaction of a person to a following traumatic event.

Studies on PTSD in regard to Cortisol levels and brain function measured salivary cortisol levels in Holocaust survivors as well as in the children of Holocaust mothers having PTSD as well as in infants of mothers with PTSD that were exposed pregnant at the 9/11 attacks on the WTC (18).

\section{RESULTS}

Consistently findings demonstrated alternated (lowered) cortisol levels in all persons with PTSD and their offspring a fact that posed the question whether these findings might be interpreted as the result of in utero changes due to traumatic stress or the result of epigenetic mechanisms. These research observations are in the direction of an epigenetic explanation of inter -individual variation and long lasting effects but have to be verified in future research. (18-20) Yehudas' actual research approach (21) is very promising as it attempts to offer a global view on gene expression and gene methylation and to specify what differentiates people with PTSD and people without PTSD in order to "identify causal networks that can help diagnose PTSD and identify new treatment targets in the brain” (22).

\section{Neurobiological findings and implications for treatment}

The most common advantage of neurobiological research on PTSD lies in the suggestion of specific pathways of medication. The established evidence based pharmaco-therapeutic treatments (23) include mostly Serotonin selective reuptake inhibitors (SSRI).

SSRI weaken several of the prominent PTSD symptoms through the enhancement of serotonergic transmission. Their efficacy for PTSD has been tested in randomized clinical trials (RCT) in comparison to placebo groups covering a bride spectrum of trauma types. Although these studies had some common methodological restrictions referring to a small sample size or high dropout rates SSRI's could be verified in the RCT with sufficient size of $\mathrm{N}=$ 208 and $\mathrm{N}=551$ as more effective in the treatment of PTSD than in placebo groups but with small effect sizes (23). These results do not apply at the same degree to veterans with long term symptoms as to recent traumatized veterans and civilian population.

Dual action serotonin norepinephrine selective reuptake inhibitors (SNRI) have been tested in just two published clinical trials involving large numbers of patients $(\mathrm{N}=329$ and $\mathrm{N}=538)$ but having high dropout rates of over $30 \%$ and concluding like SSRI's to small effect sizes (23).

Although benzodiazepines are often used as medication of choice to address anxiety and sleep disturbance, remarkably few empirical studies have been conducted in regard to their effectiveness in the treatment of PTSD.

Due to these results (23) conclude that "pharmacotherapy alone is generally not recommended, except in the circumstance when proven efficacious psychotherapies are not available" (p.343). A combination of medication and psychotherapy is suggested for the treatment of PTSD.

\section{DISCUSSION}

In regard to psychotherapy neurobiological findings can contribute decisively in the design of an appropriate treatment strategy. The clinical picture PTSD sufferers present is characterized by the disruption of experience, movement and action, the tendency to respond to triggers in the here and now with automatically activated action patterns of the traumatic past, the restricted attention capacity and working memory that causes increased engagement in the present, the difficulty to identify and articulate their sensations, feelings and physical condition in an adequate manner. Taking these facts into consideration the question emerges how can traditional approaches of CBT and psychodynamic therapy that are based on understanding and insight efficiently cope with the challenge of treating PTSD sufferers? Bessel A. Van der Kolk, a pioneer in the field of PTSD treatment concludes that "Neither CBT protocols nor psychodynamic therapeutic techniques pay sufficient attention to the experience and interpretation of disturbed physical sensations and preprogrammed physical action patterns" (24).

He pleads for the integration of techniques that address the abovementioned particularities and enable the reprogramming of automatic physical responses as follows:

1.Central for the development of an effective treatment methodology is the fact that in the experience of physical helplessness the inability or failure of the organism to activate its physiological resources and organize 
an adequate protective response ("freezing") is a crucial factor. It becomes encoded as a conditioned behavioral response and habituated over time. In reverse a first therapeutic target has to focus on provision of opportunities for the "rerouting" of the passive fear conditioning pathways to active coping strategies in the framework of "action oriented programs" (24).

2.PTSD sufferers when reminded to the traumatic event in everyday life or during verbal therapy demonstrate dys-regulated arousal of the autonomic nervous system with an increased sympathetic component and a decreased parasympathetic component. According to studies (small sample numbers) conducted by the Trauma Center of the Boston University, School of Medicine (24) Yoga promises to be an efficient technique in order to modulate arousal in objective terms but also in the subjective judgement of participants.

3.A third branch of intervention takes advantage of the PTSD neuroimaging findings in regard to the deactivation of the prefrontal cortex. PTSD sufferers are inhibited in their executive functions and encounter great difficulties in the attendance and integration of emotions, inner sensations and cognition. As they are in danger to become overwhelmed if starting meditating it is important to develop in sequences their inner capacity to observe mindfully their inner experience and sensation and their potential for introspection and self-awareness.

\section{CONCLUSION}

In practical terms, the suggested awareness-based body oriented therapy can be effective because contributes to re - establish a sense of physical control, enhance physical boundaries, uses breath and body movement in order to modify physiological arousal and focuses on assuring in a physical manner the experience of being protected (24-29).

A complex solution to a complex phenomenon? As human beings are we programmed to enhance survival through adaptation mechanisms? As scientists and clinicians we certainly have to develop methods of treating a disease as a personal experience of a global phenomenon. The traumatic experience remains a personal story to be told. Time-wise, treating the symptoms first, might be correct. Recapturing the development of such a condition through the lifespan of the individual might involve the restructuring of our idea of human evolution: Do we change as a species through traumatic events? Is PTSD the mechanism to sustain these changes? Should we intervene?

This study attempted an overview of a vast phenomenon. As the concepts of neurobiology and psychology remain interlinked, we might wish to regain our perspective in areas of research like: 1) the relation of specific bodily expressions and traumatic events as a means of survival 2) how to treat the discrepancy of the here-and-now environment with the past event 3) how to transcend the experience in the future so to deal with aggression towards others or the self..

\section{REFERENCES}

1. Howard S, Crandall M. Post-traumatic stress disorder. What happens in the brain? Washington Academy of Science. 2007;93(4):1-17.

2. American Psychiatric Association. Diagnostic and Statistical Manual of Mental Disorders 2013 (5th edn), Washington, DC: American Psychiatric Association.

3. Norr AM, Albanese BJ, Boffa JW, et al. The relationship between gender and PTSD symptoms: Anxiety sensitivity as a mechanism. Personality And Individual Differences, 2016;90:210-13.

4. Frijling JL, Olff M. Biological mechanisms of traumatic stress response, In: Figley CR. (ed) Encyclopedia of Trauma: An Interdisciplinary Guide. Thousand Oaks, Calif: SAGE Publications, Inc., 2012; 47 pp-48 pp.

5. Westphal M, Sullivan G. Neurobiological effects of trauma, in: Figley CR. (ed) Encyclopedia of trauma: An Interdisciplinary Guide. Thousand Oaks, Calif: SAGE Publications, Inc. 2012.

6. Sherin JE, Nemeroff CB. Post-traumatic stress disorder: The neurobiological impact of psychological trauma. Dialogues in Clinical Neuroscience, 2011;13(3):263-78.

7. Schore AN. The effects of early relational trauma on right brain development, affect regulation, and infant mental health. Infant Mental Health Journal, 2001;22(1-2):201-69.

8. Bowirrat A, Chen TH, Blum K, et al. Neuro-psychopharmacogenetics and neurological antecedents of posttraumatic stress disorder: Unlocking the mysteries of resilience and vulnerability. Current Neuropharmacology, 2010;8(4):335-58

9. O'Doherty DM, Chitty KM, Saddiqui S, et al. A systematic review and meta-analysis of magnetic resonance imaging measurement of structural volumes in post-traumatic stress disorder. Psychiatry Research: Neuroimaging, 2015;232(1):1-33.

10. Sartory G, Cwik J, Knuppertz H. In search of the trauma memory: A meta-analysis of functional neuroimaging studies of symptom provocation in post-traumatic stress disorder (PTSD). PLoS ONE, 2013;8(3).

11. Stark EA, Parsons CE, Van Hartevelt TJ, et al. Post-traumatic stress influences the brain even in the absence of symptoms: A systematic, quantitative meta-analysis of neuroimaging studies. Neuroscience and Bio-behavioral Reviews, 2015;56:207-21.

12. Miller GE, Chen E, Zhou ES. If it goes up, must it come down? Chronic stress and the hypothalamic-pituitary-adrenocortical axis in humans. Psychological Bulletin. 2012;133(1):25-45.

13. Yehuda R. Advances in understanding neuroendocrine alterations in PTSD and their therapeutic implications. Ann N Y Acad Sci. 2006; 1071:137-66.

14. Perroud N, Rutembesa E, Paoloni-Giacobino A, et al. The Tutsi genocide and trans-generational transmission of maternal stress: Epigenetics and biology of the HPA axis. World J Biol Psychiatry. 2014;15:334-45.

15. Yarvis JS. Transgenerational transmission of Trauma, In: Figley CR (ed) Encyclopedia of trauma: An Interdisciplinary Guide. Thousand Oaks, Calif: Sage Publications, Inc 2012; pp.671-73.

16. Dekel R, Goldblatt H. Is there intergenerational transmission of trauma? The case of combat veterans' children. American Journal of Orthopsychiatry, 2008;78(3):281-89.

17. Danieli Y, Norris FH, Engdahl B. Multigenerational legacies of trauma: Modeling the and how of transmission. American Journal of Orthopsychiatry, 2016;86(6):639-51.

18. Yehuda R, Bierer LM. The relevance of epigenetics to PTSD: Implications for the DSM-V. Journal of Traumatic Stress, 2009 a;22(5):427-34.

19. Bale TL. Epigenetic and trans-generational reprogramming of brain development. Nat Rev Neurosci. 2015;16:332-44.

20.Dias, BG, Maddox, SA, Klengel, T, et al. Epigenetic mechanisms underlying learning and the inheritance of learned behaviors. Trends Neurosci. 2015;38:96-07.

21. Yehuda R, Cai G, Golier JA, et al. Gene expression patterns associated with posttraumatic stress disorder following exposure to the World Trade Center attacks. Biological Psychiatry, 2009 b;66(7):708-711.

22. Yehuda R. Understanding PTSD and resilience, UNM Department of Psychiatry "We need to talk", assessed on 28/10/2016 in https://www. youtube.com/watch? $=$ cTdYOvcrXHs.

23. Sullivan G, Neria Y. Pharmacotherapy of PTSD: Current status and controversies. Psychiatric Annals 2009;39(6):342-47.

24.Van Der Kolk BA. Clinical implications of neuroscience research in PTSD. In R. Yehuda, R. Yehuda (Eds.), Psychobiology of post-traumatic stress disorders: A decade of progress, 2006:1071: Malden: Blackwell Publishing, UK. pp. 277-93.

25.Gapp K, Von Ziegler L, Tweedie-Cullen RY, et al. Early life epigenetic programming and transmission of stress-induced traits in mammals: How and when can environmental factors influence traits and their transgenerational inheritance? Bioessays. 2014;36:491-02.

26. Post-traumatic Stress Disorder (PTSD), 309.81 (F43.10), In: American Psychiatric Association, Diagnostic and Statistical Manual of Mental Disorders (5th edn.), Washington, DC: American Psychiatric Association, 2013(pp. $271-80$ )

27.Mahan LA, Ressler KJ. Fear conditioning, synaptic plasticity and the amygdala: Implications for posttraumatic stress disorder. Trends in Neurosciences, 2012;35(1):24-35.

28. Yehuda R. Clinical relevance of biologic findings in PTSD. Psychiatric Quarterly, 2002:73(2):123-33.

29. Yehuda R, Daskalakis NP, Bierer L, et al. Holocaust exposure induced intergenerational effects on FKBP5 methylation 2016;80(5):372-80. 